ESAIM: PROCEEDINGS, March 2013, Vol. 39, p. 66-77

M. Belhaq, P. Lafitte and T. Lelièvre Editors

\title{
QUALITATIVE PROPERTIES IN A MORE GENERAL DELAYED HEMATOPOIETIC STEM CELLS MODEL *
}

\author{
R. YAfia ${ }^{1}$ AND M. A. AzIZ-Alaoui ${ }^{2}$
}

\begin{abstract}
In this paper, we consider a more general model describing the dynamics of Hematopoietic Stem Cells (HSC) model with one delay. Its dynamics are studied in terms of local stability and Hopf bifurcation. We prove the existence of the possible steady state and their stability with respect to the time delay and without delay. We show that a sequence of Hopf bifurcations occur at the positive steady state as the delay crosses some critical values. We illustrate our results by some numerical simulations.
\end{abstract}

\section{INTRODUCTION AND MATHEMATICAL MODEL}

Hematological diseases have attracted a significant amount of modelling attention because a number of them are periodic in nature (Haurie, Dale and Mackey (1998) [18]). Some of these diseases involve only one blood cell type and are due to the destabilization of peripheral control mechanisms, e.g., periodic auto-immune hemolytic anemia (Bélair, Mackey and Mahaffy (1995) [3] ; Mahaffy, Bélair and Mackey (1998) [29]), and cyclical thrombocytopenia (Swinburne and Mackey (2000) [40]; Santillan et al. (2000) [38]). Typically, periodic hematological diseases of this type involve periodicity between two and four times the bone marrow production or maturation delay (which is different from the delay considered in this paper).

Other periodic hematological diseases involve oscillations in all of the blood cells (white cells, red cells and platelets). Examples include cyclical neutropenia (Haurie, Dale and Mackey (1999) [20]; Haurie et al. (2000) [19]) and periodic chronic myelogenous leukemia (Fortin and Mackey (1999) [14]). These diseases involve very long periodic dynamics $[15,36]$ (on the order of weeks to months) and are thought to be due to a destabilization of haematopoietic stem cells (HSC) compartment from which all of these mature blood cell types are derived, see Fowler and Mackey (2002) [15].

The population of (HSC) give rise to all of the differentiated elements of the blood : the white blood cells, red blood cells, and platelets, which may be either actively proliferating or in a resting phase. After entering the proliferating phase, a cell is committed to undergo cell division at a fixed time $\tau$ later. The generation time $\tau$ is assumed to consist of four phases, $G_{1}$ the pre-synthesis phase, $S$ the DNA synthesis phase, $G_{2}$ the post-synthesis phase and $M$ the mitotic phase.

Just after the division, both daughter cells go into the resting phase called $G_{0}$-phase. Once in this phase, they can either return to the proliferating phase and complete the cycle or die before ending the cycle. The (HSC)

\footnotetext{
* Key words and phrases : Hematopoietic Stem Cells Model; delayed differential equations ; Hopf bifurcation; periodic solutions.

${ }^{1}$ Corresponding author : R. Yafia, yafia1@yahoo.fr, Ibnou Zohr University, Polydisciplinary Faculty of Ouarzazate, B.P : 638, Ouarzazate, Morocco

2 Laboratoire de Mathématiques Appliquées, 25 Rue Ph. Lebon, BP 540, 76058 Le Havre Cedex, France. aziz.alaoui@univlehavre.fr

(C) EDP Sciences, SMAI 2013
} 
model that we consider is a classical $G_{0}$ model, see $[9,26,39]$ and reference therein.

The full model for this situation consists of a pair of (age structured) reaction convection evolution equations with their associated boundary and initial conditions [22,23, 27,37].

Using the method of characteristics [45] these equations can be transformed into a pair of non-linear first order differential delay equations, see $[1,15,22,24]$, [25], see also [36] and references therein cited,

$$
\left\{\begin{array}{c}
\frac{d N}{d t}=-\delta N-\beta(N) N+2 e^{-\gamma \tau} \beta\left(N_{\tau}\right) N_{\tau} \\
\frac{d P}{d t}=-\gamma P+\beta(N) N-e^{-\gamma \tau} \beta\left(N_{\tau}\right) N_{\tau}
\end{array}\right.
$$

where $\beta$ is a monotone decreasing function of $N$ which has the explicit form of a Hill function (see $[8,13,22$, 33]) :

$$
\beta(N)=\beta_{0} \frac{\theta^{n}}{\theta^{n}+N^{n}}
$$

The symbols in equation (1) have the following interpretation. $N$ is the number of cells in non-proliferative phase, $N_{\tau}=N(t-\tau), P$ the number of cycling proliferating cells, $\gamma$ the rate of cells loss from proliferative phase, $\delta$ the rate of cells loss from non-proliferative phase, $\tau$ the time spent in the proliferative phase, $\beta$ the feedback function, rate of recruitment from non-proliferative phase, $\beta_{0}>0$ the maximal rate of re-entry in the proliferating phase, $\theta \geq 0$ is the number of resting cells at which $\beta$ has its maximum rate of change with respect to the resting phase population, $n>0$ describes the sensitivity of reintroduction rate with changes in the population, and $e^{-\gamma \tau}$ accounts for the attenuation due to apoptosis (programmed cell death) at rate $\gamma$.

The model (1) was intensively studied by many authors, see for example, $[1,6,7,13,15,22-25,35,36,41-43]$, this list being not exhaustive.

For numerical study, typical values of the parameters for humans are given by Mackey (1978), (1997) $[22,24]$ as

$$
\delta=0.05 d^{-1}, \quad \beta_{0}=1.77 d^{-1}, \quad \tau=2.2 d, \quad n=3 .
$$

(The value of $\theta$ is $1.62 \times 10^{8}$ cells $\mathrm{Kg}^{-1}$, but this is immaterial for dynamic considerations). For values of $\gamma$ in the range $0.2 d^{-1}$, the consequent steady state is unstable and there is a periodic solution whose period $T$ at the bifurcation ranges from 20 - 40 days, see (Fowler and Mackey, 2002) [15]. In [22,23] the author proves that the stability of the non trivial steady state depend on the value of $\gamma$. When $\gamma=0$, this steady state cannot be destabilized to produce dynamics characteristic of periodic hematopoiesis. On the other hand, for $\gamma>0$, increase in $\gamma$ lead to a decrease in the (HSC) numbers and a consequent decrease in the cellular efflux (given by $\delta N$ ) into the differentiated cell lines. This diminished efflux becomes unstable when a critical value of $\gamma$ is reached, $\gamma=\gamma_{1}$, at which a supercritical Hopf bifurcation occurs. For all values of $\gamma$ satisfying $\gamma_{1}<\gamma<\gamma_{2}$, there is a periodic solution of the above model whose period is in good agreement with that seen in periodic hematopoiesis. At $\gamma=\gamma_{2}$, a reverse bifurcation occurs and greatly diminished (HSC) numbers as well as cellular efflux again become unstable.

In [35], authors numerically investigate the influence of each parameter $\left(\tau, \delta, \gamma, \beta_{0}\right.$ and $\left.n\right)$ on the oscillation characteristics, see [36]. In [36], authors consider the limiting case $(n=+\infty)$ of the above model in order to compute an explicit solution, give an exact form of the period and the amplitude of oscillations. They illustrate these results numerically and show that the main parameters controlling the period are $\left(\tau, \delta, \gamma, \beta_{0}\right.$ and $\left.n\right)$ mainly influence the amplitude. These authors consider $n=12$ as a good approximation of high Hill coefficient for their numerical simulations. The Hill coefficient $n$ is often regarded as a cooperativity coefficient, describing the number of agents (molecules, proteins or complexes) required to activate or deactivate a given process. If $n$ was interpreted to be the number of ligand molecules required to active or deactivate a receptor site, then values of $n=12$ or larger would not biologically realistic. However, there are other situations in which cascade effects are known to create switch like phenomena [13]. In these circumstances, both experimental data and theoretical modelling suggest that the large values of $n$ considered are quite realistic $[6,7,36]$.

It is generally believed that normal and malignant cell population have different cell cycle times (Andersen and 
Mackey (2000) [1], Baserga (1981) [2]) and thus they will be described by different parameters in the above model. In particular, in untreated leukemic cells the apoptotic rate $\gamma$ is significantly smaller than in normal cells (Macnamara et al., (1999) [28] ; Okita et al., (2000) [31]; Ong et al., (2000) [32]; Parker et al., (2000) [34]), and the time spent $\tau$ in the proliferating phase is longer relative to normal cells in the bone morrow, see also (Andersen and Mackey 2001) [1].

In this paper, we are interested on the more general mathematical model of (1) given by the following system of delay differential equations by replacing the quantity $e^{-\gamma \tau}$ by the Lasota function $e^{-\gamma N_{\tau}}$ see Wazewska and Lasota 1976 [44]; it measures the production rate of proliferating hematopoietic stem cells between $t-\tau$ and $t$ :

$$
\left\{\begin{array}{c}
\frac{d N}{d t}=-\delta N-\beta(N) N+2 e^{-\gamma N_{\tau}} \beta\left(N_{\tau}\right) N_{\tau} \\
\frac{d P}{d t}=-\gamma P+\beta(N) N-e^{-\gamma N_{\tau}} \beta\left(N_{\tau}\right) N_{\tau}
\end{array}\right.
$$

The idea comes from the paper of Wazewska and Lasota 1976 [44], in which the authors presented a model of the erythropoietic (red blood cells) system in the form of one autonomous functional differential equation :

$$
\frac{d u(t)}{d t}=-\sigma u(t)+\rho e^{-\gamma u(t-H)}
$$

$\sigma>0, \rho>0, \gamma>0, H>0$.

where $u(t)$ is the red blood cell number at $t \geq 0, \sigma$ the rate of the red blood cells, $\rho$ and $\gamma$ de $\gamma$ describe the production of the red blood cells per unite time and $H$ is the time required to produce a red blood cell.

We show that, under some natural assumptions, the non-trivial fixed state is stable for small delay times, and later it looses the stability via a supercritical Hopf bifurcation, giving birth to a stable periodic solution.

This paper is organized as follows. In section 1, we prove the existence and stability of the possible steady states with/without delay. Section 2 is devoted to the occurrence of Hopf bifurcation by considering the delay as a parameter bifurcation, we prove the occurrence of a sequence of Hopf bifurcation. In the end we illustrate our result by a numerical simulations.

\section{Steady states and stability}

\subsection{Existence of possible steady states}

Consider the system :

$$
\left\{\begin{array}{c}
\frac{d N}{d t}=-\delta N-\beta(N) N+2 e^{-\gamma N_{\tau}} \beta\left(N_{\tau}\right) N_{\tau} \\
\frac{d P}{d t}=-\gamma P+\beta(N) N-e^{-\gamma N_{\tau}} \beta\left(N_{\tau}\right) N_{\tau}
\end{array}\right.
$$

The equilibrium points are given be resolving the equations

$$
\left\{\begin{array}{l}
\frac{d N}{d t}=0 \\
\frac{d P}{d t}=0
\end{array}\right.
$$

Let $d=\frac{\ln (2)}{\gamma}$ and define the function $F(N)=\beta(N)\left(2 e^{-\gamma N}-1\right)$.

As $F(0)=\beta_{0}$ and $F(d)=0$, we have that $F$ is a positive decreasing function on $] 0, d[$

From equation $(6)_{1}$, there exits $\left.N^{*} \in\right] 0, d\left[\right.$ such that $F\left(N^{*}\right)=\delta$ iff $\left.\delta \in\right] 0, \beta_{0}[$.

Where $N^{*}=F^{-1}(\delta)$, and from the equation $(6)_{2}$ we obtain that

$$
P^{*}=\frac{1}{\gamma}\left(1-e^{-\gamma N^{*}}\right) \beta\left(N^{*}\right) N^{*}
$$

Then, we have the following result 
Proposition 1.1. 1) If $\delta>\beta_{0}$, system (5) have a unique trivial equilibrium point $E_{0}=(0,0)$.

2) If $\delta \in] 0, \beta_{0}\left[\right.$, system (5) have two equilibrium points the first is trivial $E_{0}=(0,0)$ and the second is non trivial (positive) given by $E^{*}=\left(N^{*}, P^{*}\right)$, where $N^{*}=F^{-1}(\delta)$ and $P^{*}=\frac{1}{\gamma}\left(1-e^{-\gamma N^{*}}\right) \beta\left(N^{*}\right) N^{*}$.

\subsection{Stability of steady states for $\tau=0$}

For $\tau=0$ system (5) becomes a system of ordinary differential equations given by the following system :

$$
\left\{\begin{array}{c}
\frac{d N}{d t}=-\delta N-\beta(N) N+2 e^{-\gamma N} \beta(N) N \\
\frac{d P}{d t}=-\gamma P+\beta(N) N-e^{-\gamma N} \beta(N) N
\end{array}\right.
$$

Proposition 1.2. 1) If $\delta>\beta_{0}$, the trivial equilibrium point $E_{0}=(0,0)$ is asymptotically stable.

2) If $\delta \in] 0, \beta_{0}\left[\right.$, the equilibrium point $E_{0}=(0,0)$ is unstable and the non trivial (positive) $E^{*}=\left(N^{*}, P^{*}\right)$ is asymptotically stable.

Démonstration. 1) The steady states are the same given in Proposition 1.1. The stability of $E_{0}=(0,0)$, we linearize system (7) around the concerned steady state $E_{0}$.

The linearized equation is given as follows :

$$
\left\{\begin{array}{c}
\frac{d N}{d t}=-\delta N+\beta(0) N \\
\frac{d P}{d t}=-\gamma P
\end{array}\right.
$$

and the characteristic equation associated to $E_{0}$ :

$$
(\lambda+\delta-\beta(0))(\lambda+\gamma)=0
$$

Then, the characteristic roots are as follow $\lambda_{1}=-\gamma$ and $\lambda_{2}=-\delta+\beta_{0}$.

2) Suppose now that $0<\delta<\beta_{0}$ and let $N=x+N^{*}$ and $P=y+P^{*}$.

We linearize the system $(7)$ around the equilibrium point $E^{*}$ and the linearized system is given as follows :

$$
\left\{\begin{array}{c}
\frac{d x}{d t}=-\delta x+\left\{-\beta^{\prime}\left(N^{*}\right) N^{*}-2 \gamma e^{-\gamma N^{*}} \beta\left(N^{*}\right) N^{*}+2 e^{-\gamma N^{*}} \beta^{\prime}\left(N^{*}\right) N^{*}\right\} x \\
\frac{d y}{d t}=-\gamma y+\left\{\gamma \frac{P^{*}}{N^{*}}+\gamma \frac{P^{*} \beta^{\prime}\left(N^{*}\right)}{\beta\left(N^{*}\right)}+\gamma e^{-\gamma N^{*}} \beta\left(N^{*}\right) N^{*}\right\} x
\end{array}\right.
$$

The characteristic equation is given as follows

$$
(\lambda+\gamma)\left(\lambda+\beta^{\prime}\left(N^{*}\right) N^{*}+2 \gamma e^{-\gamma N^{*}} \beta\left(N^{*}\right) N^{*}-2 e^{-\gamma N^{*}} \beta^{\prime}\left(N^{*}\right) N^{*}\right)=0
$$

and the associated characteristic roots are as follow : $\lambda_{1}=\left(2 e^{-\gamma N^{*}}-1\right) \beta^{\prime}\left(N^{*}\right) N^{*}-2 \gamma e^{-\gamma N^{*}} \beta\left(N^{*}\right) N^{*}$ and $\lambda_{2}=-\gamma$. As $\beta$ is a decreasing positive function and $2 e^{-\gamma N^{*}}-1>0$, we have $\lambda_{i}<0, i=1,2$.

Then the steady states $E^{*}$ is asymptotically stable.

\subsection{Stability of steady states for $\tau>0$}

Proposition 1.3. If $\delta>\beta_{0}$, the trivial equilibrium point $E_{0}=(0,0)$ is asymptotically stable for all $\tau>0$.

2) If $\delta \in] 0, \beta_{0}\left[\right.$ and $\beta_{0}<2$ and $N^{*}<\inf \left(d=\frac{\ln (2)}{\gamma},\left(\frac{\gamma}{2}\right)^{\frac{1}{n-1}}\right)$, there exist $\tau_{0}>0$, such that the non trivial (positive)steady state $E^{*}=\left(N^{*}, P^{*}\right)$ is asymptotically for $\tau<\tau_{0}$ and unstable for $\tau>\tau_{0}$ and the equilibrium point $E_{0}=(0,0)$ is unstable for all $\tau>0$. 
Démonstration. 1) By linearizing system (3) around the steady state $E_{0}$, we obtain the following linearized equation

The characteristic equation is :

$$
\left\{\begin{array}{c}
\frac{d N}{d t}=-\delta N-\beta_{0} N+2 \beta_{0} N_{\tau} \\
\frac{d P}{d t}=-\gamma P+\beta_{0} N-\beta_{0} N_{\tau}
\end{array}\right.
$$

$$
(\lambda+\gamma)\left(\lambda+\delta+\beta_{0}-2 \beta_{0} e^{-\lambda \tau}\right)=0
$$

For the stability of $E_{0}$, one need to study the position of characteristic roots of the following equation

$$
\left(\lambda+\delta+\beta_{0}-2 \beta_{0} e^{-\lambda \tau}\right)=0 .
$$

From the proposition $1.3, E_{0}$ is asymptotically stable. For the change of stability, replacing $\lambda=i \omega$ in (14) and by separating the real and imaginary parts we have

$$
\left\{\begin{array}{c}
\delta+\beta_{0}-2 \beta_{0} \cos (\omega \tau)=0 \\
\omega+2 \beta_{0} \sin (\omega \tau)=0
\end{array}\right.
$$

From (15), we have $\omega^{2}=\left(\beta_{0}-\delta\right)\left(3 \beta_{0}+\delta\right)$. As $\beta_{0}<\delta$ there exit any value of $\tau$ in which $E_{0}$ changes the stability. Then, we conclude that $E_{0}$ is asymptotically stable for all $\tau>0$.

2) Suppose now that $\tau>0$ and $\delta<\beta_{0}$, and by linearizing the system (3) around the non trivial steady state we have the following linearized system

$$
\left\{\begin{array}{c}
\frac{d x(t)}{d t}=-\delta x(t)-h\left(N^{*}\right) x(t)+2 g\left(N^{*}\right) x(t-\tau) \\
\frac{d y(t)}{d t}=-\gamma y(t)+h\left(N^{*}\right) x(t)-g\left(N^{*}\right) x(t-\tau)
\end{array}\right.
$$

where

$$
h\left(N^{*}\right)=\beta\left(N^{*}\right)+\beta^{\prime}\left(N^{*}\right) N^{*}=(\beta(N) N)_{N=N^{*}}^{\prime}
$$

and

and

$$
g\left(N^{*}\right)=e^{-\gamma N^{*}} \beta\left(N^{*}\right)-\gamma e^{-\gamma N^{*}} \beta\left(N^{*}\right) N^{*}+e^{-\gamma N^{*}} \beta^{\prime}\left(N^{*}\right) N^{*}=\left(e^{-\gamma N} \beta(N) N\right)_{N=N^{*}}^{\prime}
$$

The characteristic equation is

$$
x=N-N^{*} \quad y=P-P^{*} .
$$

$$
\Delta(\lambda, \tau)=(\lambda+\gamma)\left(\lambda+\delta+h\left(N^{*}\right)-2 g\left(N^{*}\right) e^{-\lambda \tau}\right)=0
$$

To study the change of stability, replacing $\lambda=i \omega$ and separating the real and imaginary parts we have $\delta+$ $h\left(N^{*}\right)-2 g\left(N^{*}\right) \cos (\omega \tau)=0$ and $\omega+g\left(N^{*}\right) \sin (\omega \tau)=0$.

Then

$$
\omega^{2}=4 g\left(N^{*}\right)^{2}-\left(\delta+h\left(N^{*}\right)\right)^{2}=\left(2 g\left(N^{*}\right)-\delta-h\left(N^{*}\right)\right)\left(2 g\left(N^{*}\right)+\delta+h\left(N^{*}\right)\right)
$$

From the expressions of $h$ and $g$, we have

$$
2 g\left(N^{*}\right)-\delta-h\left(N^{*}\right)=\left(2 e^{-\gamma N^{*}}-1\right) \beta^{\prime}\left(N^{*}\right) N^{*}-2 \gamma e^{-\gamma N^{*}} \beta\left(N^{*}\right) N^{*}<0
$$

By calculations, we obtain :

$$
2 g\left(N^{*}\right)+\delta+h\left(N^{*}\right)=2 e^{-\gamma N^{*}} \beta\left(N^{*}\right)\left(2-\gamma N^{*}\right)+\beta^{\prime}\left(N^{*}\right) N^{*}+2 e^{-\gamma N^{*}} \beta^{\prime}\left(N^{*}\right) N^{*} .
$$


From the expression of $\beta$, we have

$$
\begin{aligned}
2 e^{-\gamma N^{*}} \beta\left(N^{*}\right)\left(2-\gamma N^{*}\right)+2 e^{-\gamma N^{*}} \beta^{\prime}\left(N^{*}\right) N^{*} & =2 e^{-\gamma N^{*}} \beta\left(N^{*}\right)\left(2-\gamma N^{*}-\frac{\beta_{0} \theta^{n}}{\theta^{n}+N^{* n}}\right) \\
& =2 e^{-\gamma N^{*}} \beta\left(N^{*}\right)\left(2 N^{* n}-\gamma N^{*}+\left(2-\beta_{0}\right) \theta^{n}\right)
\end{aligned}
$$

As $\beta_{0}<2$ and $N^{*}<\inf \left(\frac{\ln (2)}{2},\left(\frac{\gamma}{2}\right)^{\frac{1}{n-1}}\right)$ and from the expression of the function $\beta$, we have :

$$
g\left(N^{*}\right)+\delta+h\left(N^{*}\right)<0
$$

and the quantity of $\omega^{2}$ is positive.

As

let

$$
\left|\frac{\delta+h\left(N^{*}\right)}{g\left(N^{*}\right)}\right|<1
$$

and

$$
\tau_{k}=\frac{1}{\omega_{0}}\left\{\arccos \left(\frac{\delta+h\left(N^{*}\right)}{g\left(N^{*}\right)}\right)+2 k \pi\right\}, k=0,1,2,3, \ldots,
$$

$$
\omega_{0}=\sqrt{g\left(N^{*}\right)^{2}-\left(\delta+h\left(N^{*}\right)\right)^{2}}
$$

Then equation (17) has a pair of purely imaginary roots $\pm i \omega_{0}$ at $\tau=\tau_{k}, k=0,1,2,3, \ldots$.

Let $\lambda(\tau)=\eta(\tau)+\omega(\tau)$ denote a root of (17) near $\tau=\tau_{k}$, such that $\eta\left(\tau_{k}\right)=0, \omega\left(\tau_{k}\right)=\omega_{0}$.

Then, we deduce the result.

\section{Periodic SOlUtions AND NUMERICAL SIMUlations}

We apply Hopf bifurcation theorem to show the existence of nontrivial periodic solution of system (5), for suitable values of parameter delay, used as a bifurcation parameter. Therefore, the periodicity is a result of changing the type of stability, from stable stationary solution to limit cycle.

In what follows, we recall the formulation of the Hopf bifurcation Theorem for retarded differential equations. Let the equation

$$
\frac{d x(t)}{d t}=F\left(\alpha, x_{t}\right)
$$

with $F: \mathbb{R} \times C \longrightarrow \mathbb{R}^{n}, F$ of class $\mathcal{C}^{k}, k \geq 2$ and $F(\alpha, 0)=$ ofor all $\alpha \in \mathbb{R}$ and $C=C\left([-r, 0], \mathbb{R}^{n}\right)$ the space of continuous functions from $[-r, 0]$ into $\mathbb{R}^{n}$. As usual, $x_{t}$ is the function defined from $[-r, 0]$ into $\mathbb{R}^{n}$ by $x_{t}(\theta)=x(t+\theta), r \geq 0$ and $n \in \mathbb{N}^{*}$.

The following assumptions are stated :

$\left(M_{0}\right) F$ of class $\mathcal{C}^{k}, k \geq 2$ and $F(\alpha, 0)=0$ for all $\alpha \in \mathbb{R}$, and the map $(\alpha, \varphi) \longrightarrow D_{\varphi}^{k} F(\alpha, \varphi)$ sends bounded sets into bounded sets.

$\left(M_{1}\right)$ The characteristic equation

$$
\Delta(\alpha, \lambda)=\operatorname{det}\left(\lambda I d-D_{\varphi} F(\alpha, 0) \exp (\lambda(.) I d)\right),
$$

of the linearized equation of (20) around the equilibrium $v=0$ :

$$
\frac{d v(t)}{d t}=D_{\varphi} F(\alpha, 0) v_{t}
$$

has in $\alpha=\alpha_{0}$ a simple imaginary root $\lambda_{0}=\lambda\left(\alpha_{0}\right)=i$, all others roots $\lambda$ satisfy $\lambda \neq m \lambda_{0}$ for $m \in \mathbb{Z}$. 
$\left(M_{2}\right) \lambda(\alpha)$ being the branch of roots passing through $\lambda_{0}$, we have

$$
\frac{\partial}{\partial \alpha} \operatorname{Re} \lambda(\alpha)_{/ \alpha=\alpha_{0}} \neq 0
$$

Théorème 2.1. [16] Under the assumptions $\left(M_{0}\right),\left(M_{1}\right)$ and $\left(M_{2}\right)$, there exist constants $\varepsilon_{0}>0$ and $\delta_{0}$ and functions $\alpha(\varepsilon), T(\varepsilon)$ and a $T(\varepsilon)$-periodic function $x^{*}(\varepsilon)$, such that :

a) All of these functions are of class $\mathcal{C}^{k-1}$ with respect to $\varepsilon$, for $\varepsilon \in\left[0, \varepsilon_{0}\left[, \alpha(0)=\alpha_{0}, T(0)=2 \pi, x^{*}(0)=0\right.\right.$;

b) $x^{*}(\varepsilon)$ is a $T(\varepsilon)$-periodic solution of $(20)$, for the parameter values equal $\alpha(\varepsilon)$;

c) For $\left|\alpha-\alpha_{0}\right|<\delta_{0}$ and $|T-2 \pi|<\delta_{0}$, any $T$-periodic solution $p$, with $\|p\|<\delta_{0}$, of (20) for the parameter value $\alpha$, there exists $\varepsilon \in\left[0, \varepsilon_{0}\left[\right.\right.$ such that $\alpha=\alpha(\varepsilon), T=T(\varepsilon)$ and $p$ is up to a phase shift equal to $x^{*}(\varepsilon)$.

Normalizing the delay $\tau$ by the time scaling $t \rightarrow \frac{t}{\tau}$, effecting the change of variables $u(t)=N(t \tau)$ and $v(t)=P(t \tau)$, the system (3) is transformed into

$$
\left\{\begin{array}{c}
\dot{u}(t)=\tau\left[-\delta u(t)-\alpha(u(t))+2 e^{-\gamma u(t-1)} \alpha(u(t-1))\right] \\
\dot{v}(t)=\tau\left[-\gamma v(t)+\alpha(u(t))-e^{-\gamma u(t-1)} \alpha(u(t-1))\right]
\end{array}\right.
$$

where $\alpha(x)=\beta(x) x$

By the translation $z(t)=(u(t), v(t))-\left(N^{*}, P^{*}\right)$, system $(24)$ is written as a functional differential equation $(\mathrm{FDE})$ in $C:=C\left([-1,0], \mathbb{R}^{2}\right)$ :

$$
\dot{z}(t)=L(\tau) z_{t}+f\left(z_{t}, \tau\right)
$$

where $L(\tau): C \longrightarrow \mathbb{R}^{2}$ is a linear operator and $f_{0}: C \times \mathbb{R} \longrightarrow \mathbb{R}^{2}$ are given respectively by :

$$
\begin{gathered}
L(\tau) \varphi=\tau\left(\begin{array}{c}
-\left(\delta+\alpha^{\prime}\left(N^{*}\right) \varphi_{1}(0)+\left(-2 \gamma e^{-\gamma N^{*}} \alpha\left(N^{*}\right)+2 e^{-\gamma N^{*}} \alpha^{\prime}\left(N^{*}\right)\right) \varphi_{1}(-1)\right. \\
-\gamma \varphi_{2}(0)+\alpha^{\prime}\left(N^{*}\right) \varphi_{1}(0)-\left(-\gamma e^{-\gamma N^{*}} \alpha\left(N^{*}\right)+e^{-\gamma N^{*}} \alpha^{\prime}\left(N^{*}\right)\right) \varphi_{1}(-1)
\end{array}\right) \\
f(\varphi, \tau)=\tau\left(\begin{array}{c}
-\alpha\left(\varphi_{1}(0)+N^{*}\right)+\alpha^{\prime}\left(N^{*}\right) \varphi_{1}(0)+2 e^{-\gamma\left(\varphi_{1}(-1)+N^{*}\right)} \alpha\left(\varphi_{1}(-1)+N^{*}\right)-\delta N^{*} \\
+\left(2 \gamma e^{-\gamma\left(\varphi_{1}(-1)+N^{*}\right)} \alpha\left(N^{*}\right)-2 e^{-\gamma\left(\varphi_{1}(-1)+N^{*}\right)} \alpha^{\prime}\left(N^{*}\right)\right) \varphi_{1}(-1) \\
\alpha\left(\varphi_{1}(0)+N^{*}\right)-\alpha^{\prime}\left(N^{*}\right) \varphi_{1}(0)-e^{-\gamma\left(\varphi_{1}(-1)+N^{*}\right)} \alpha\left(\varphi_{1}(-1)+N^{*}\right)-\gamma P^{*} \\
+\left(-\gamma e^{-\gamma\left(\varphi_{1}(-1)+N^{*}\right)} \alpha\left(N^{*}\right)+e^{-\gamma\left(\varphi_{1}(-1)+N^{*}\right)} \alpha^{\prime}\left(N^{*}\right)\right) \varphi_{1}(-1) .
\end{array}\right)
\end{gathered}
$$

for $\varphi=\left(\varphi_{1}, \varphi_{2}\right) \in C$.

The following theorem gives the existence of bifurcating periodic solutions.

Théorème 2.2. Suppose that $\delta \in] 0, \beta_{0}\left[, \beta_{0}<2\right.$ and $N^{*}<\inf \left(\frac{\ln (2)}{2},\left(\frac{\gamma}{2}\right)^{\frac{1}{n-1}}\right)$, for each $k \in \mathbb{N}$ and $\tau$ in a neighborhood of $\tau_{k}$, equation (24) has a family of periodic solutions $p_{k}(\varepsilon)$ with period $T_{k}=T_{k}(\varepsilon)$, for the parameter values $\tau=\tau_{k}(\varepsilon)$ such that $p_{k}(0)=0\left(p_{k}(0)=\left(N^{*}, P^{*}\right)\right.$ for the system $\left.(3)\right), T_{k}(0)=\frac{2 \pi}{\omega_{0}}$ and $\tau_{k}(0)=\tau_{k}$. Where $\tau_{k}, k=0,1,2, \ldots$ and $\omega_{0}$ are given respectively in equations (18) and (19).

Démonstration. We apply the Hopf bifurcation theorem. From the expression of $f$ in (25), we have,

$$
f(0, \tau)=0 \quad \text { and } \quad \frac{\partial f(0, \tau)}{\partial \varphi}=0, \text { for all } \tau>0
$$

From (17), we have :

$$
\Delta(i \omega, \tau)=0 \quad \Leftrightarrow \quad\left\{\begin{array}{c}
\omega=\omega_{0} \\
\text { and } \\
\tau=\tau_{k}, k=0,1,2, \ldots
\end{array}\right.
$$




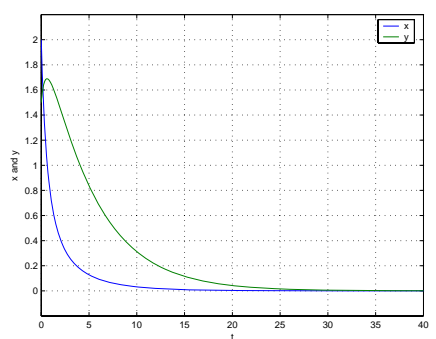

Figure 1. Stability of $E_{0}=(0,0)$ and the nonexistence of $E^{*}$ for $\delta>\beta_{0}$

Thus, characteristic equation (17) has a pair of simple imaginary roots $\lambda_{0}=i \omega_{0}$ and $\bar{\lambda}_{0}=-i \omega_{0}$ at $\tau=\tau_{k}$, $k=0,1,2, \ldots$.

Lastly, we need to verify the transversality condition.

From (17), $\Delta\left(\lambda_{0}, \tau_{k}\right)=0$ and $\frac{\partial}{\partial \lambda} \Delta\left(\lambda_{0}, \tau_{k}\right)=\left(\lambda_{0}+\gamma\right)\left(1-\tau_{k} g\left(N^{*}\right) e^{-\lambda \tau_{k}}\right) \neq 0$. According to the implicit function theorem, there exists a complex function $\lambda=\lambda(\tau)$ defined in a neighborhood of $\tau_{k}$, such that $\lambda\left(\tau_{k}\right)=\lambda_{0}$ and $\Delta(\lambda(\tau), \tau)=0$ and

$$
\lambda^{\prime}(\tau)=-\frac{\partial \Delta(\lambda, \tau) / \partial \tau}{\partial \Delta(\lambda, \tau) / \partial \lambda}, \text { for } \tau \text { in a neighborhood of } \tau_{k}, k=0,1,2, \ldots
$$

Let $\lambda(\tau)=\eta(\tau)+\omega(\tau)$. From (26) we have :

$$
\eta^{\prime}(\tau)_{/ \tau=\tau_{k}}=\frac{2 \omega_{0}^{2}}{\left(1+2 \tau_{k} g\left(N^{*}\right) \cos \left(\omega_{0} \tau_{k}\right)\right)^{2}+\left(2 \tau_{k} g\left(N^{*}\right) \sin \left(\omega_{0} \tau_{k}\right)\right)^{2}} \text { for } k=0,1,2, \ldots
$$

By the continuity property, we conclude that, $\eta^{\prime}(\tau)_{/ \tau=\tau_{k}}>0$, for $k=0,1,2, \ldots$.

Remarque 2.3. In Theorem 2.2, we have proved that the system (24) has a sequence of Hopf bifurcation. We prove that the first derivative of real part $\eta(\tau)$ at $\tau=\tau_{k}, k=0,1,2, \ldots$ is strictly positive. To prove that the bifurcation is subcritical or supercritical, we need to calculate the second derivative of $\tau$ at 0 , such that $\tau(0)=\tau_{k}$ for $\tau$ in a neighborhood of $\tau_{k}, k=0,1,2, \ldots$

If $\tau^{\prime \prime}(0)>0$ the bifurcation is supercritical and the bifurcating branch of periodic solutions are stable and if $\tau^{\prime \prime}(0)<0$ the bifurcation is subcritical and the bifurcating branch of periodic solutions are unstable.

With the aid of Theorem 2.1, it is also straightforward to check for possible Hopf bifurcations as we increase the delay $\tau$. The importance of Hopf bifurcations in this context is that at the bifurcation point a limit cycle is formed around the fixed point. The existence of periodic solutions is relevant in haematopoiesis models, because it implies that the number of normal or malignant stem cells levels may oscillate at different time of the delay around a fixed point even in the absence of any treatment. Such a phenomenon has been observed clinically $[1,24,36]$. The result presented here support the hypothesis that oscillations in dynamical hematological diseases $[1,24,36]$ are driven by oscillations in the stem cell compartment. We find that oscillations consistent with those observed for cyclical neutropenia are initiated when an increase in demand for circulating blood cells causes the stem cell compartment to undergo a sequence of Hopf bifurcation.

Neutrophil numbers reach dangerously low levels, oscillating with a period of 19-21 days in humans and 11-16 days in dogs. Longer periods of up to 50 days have been observed [10,18]. Platelet levels oscillate around their mean value with the same period. In periodic chronic myelogenous leukemia, leukocyte levels oscillate far above normal values with very long periods, ranging from 40 - 80 days $[10,14]$. 


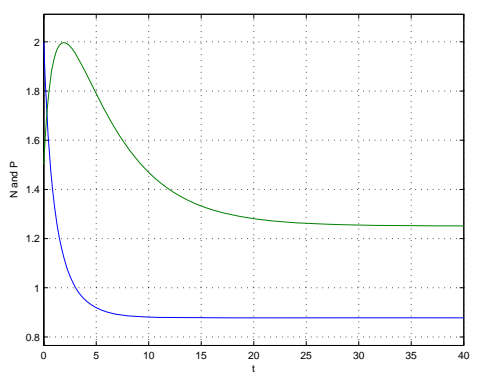

FiguRE 2. Instability of $E_{0}=(0,0)$ and stability of $E^{*}$ for $\tau=0$ and $\delta<\beta_{0}$

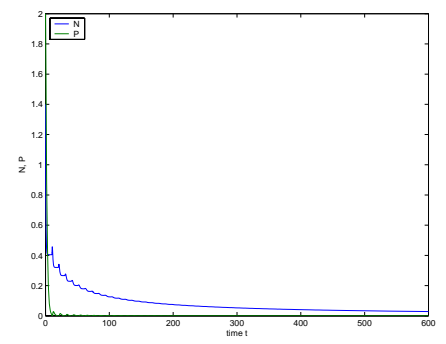

Figure 3. Stability of $E_{0}$ for $\tau>0$

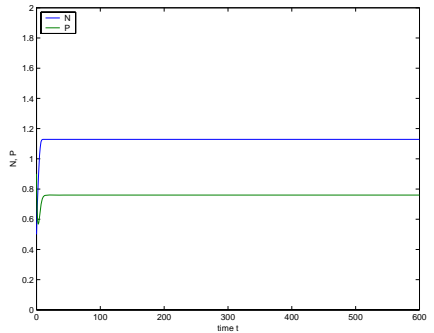

Figure 4. Instability of $E_{0}$ and stability of $E^{*}$ for $\tau<\tau_{0}$

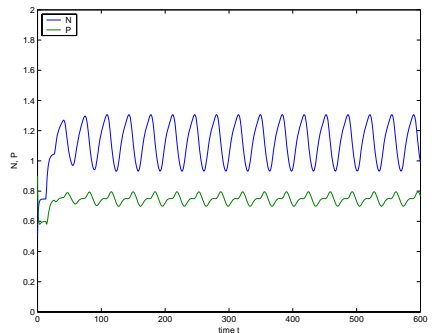

Figure 5. Periodic solutions around the bifurcating point $E^{*}$ for $\tau=\tau_{0}$ 


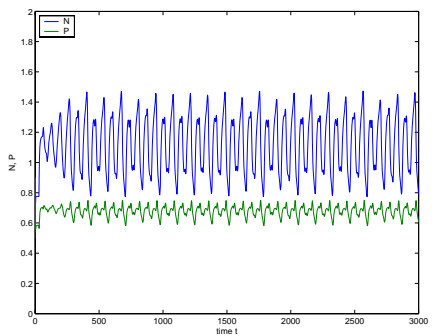

Figure 6. Periodic solutions around the bifurcating point $E^{*}$ for $\tau=\tau_{1}$

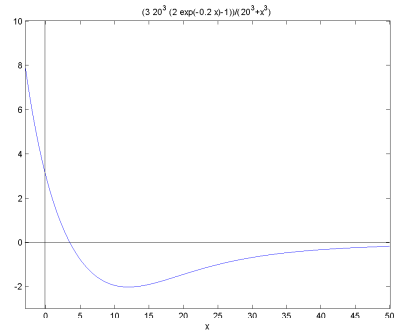

Figure 7. The curve of the functional $F$ showing the existence of $N^{*}$.
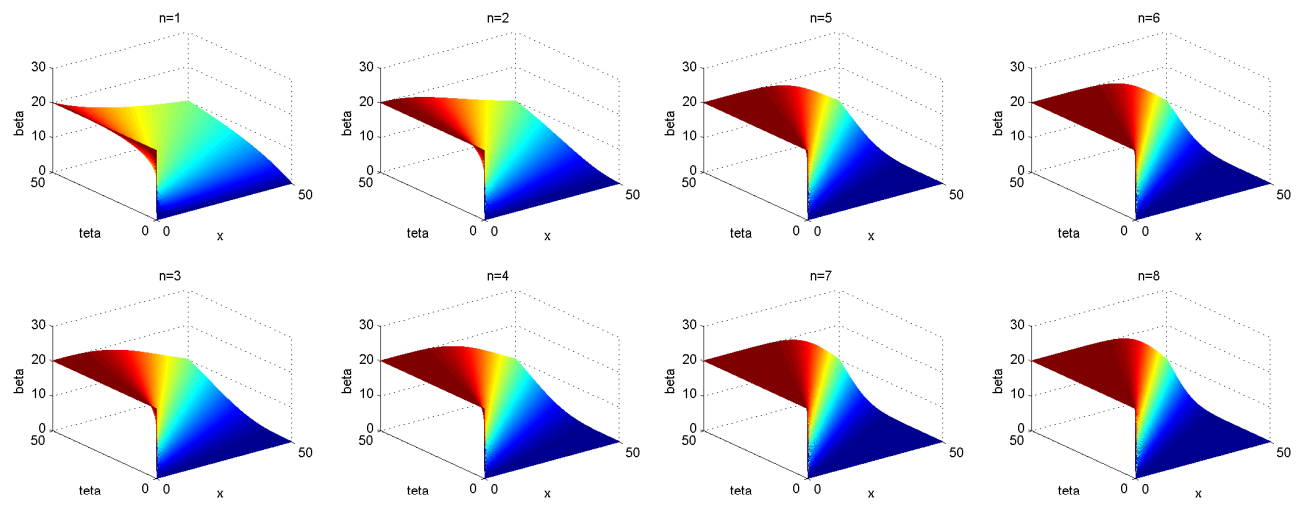

Figure 8 . The curve of the functional $\beta$ with respect to $x$ and $\theta$.

\section{ACKNOWLEDGEMENT}

We thank the Professor M.C. Mackey for encouragement and helpful discussions. We would like, also to thank the referees and the editor for their careful reading of the original manuscript and their many valuable comments and suggestions that greatly improved the presentation of this work.

\section{REFERENCES}

[1] L. K. Andersen and M. C. Mackey, Resonance in Periodic Chemotherapy : A Case Study of Acute Myelogenous Leukemia. J. theor. Biol. 209 (2001) 113-130.

[2] R. Baserga, The cell cycle, New Engl. J. Med. 304 (1981) 453-459.

[3] J. Bélair, J. M. Mahaffy and M. C. Mackey, Age structured and two delay models for erythropoiesis, Math. Biosc. 128 (1995) 317-346. 
[4] E. Beretta and Y. Kuang, Geometric stability switch criteria in delay differential systems with delay dependent parameters, SIAM J. Math. Anal., Vol. 33 No. 5 (2002) 1144-1165.

[5] S. Bernard and J. Bélair and M. C. Mackey, Sufficient conditions for stability of linear differential equations with distributed delay, Discrete and Continuous Dynamical Systems Series B, Vol. 1, No. 2 (2001) 233-256.

[6] S. Bernard, B. Cajavec, L. Pujo-Menjouet, M. C. Mackey and H. Herzel, Modeling transcriptional feedback loops : The role of Gro/TLE1 in Hes1 oscillations, submitted to Biophys. J. (2004).

[7] H. Bolouri and E. H. Davidson, Transcriptional regulatory cascades in developemnt : Initial rates, not steady state, determine network kinetics, Proc. Natl. Acad. Sci. USA 100 (2003) 9371-9376.

[8] W. S. Bullough, Mitotic control in adult mammalian tissues, Biol. Rev. 50 (1975) 99-127.

[9] F. Burns and I. Tannock, On the existence of a $G_{0}$ phase in the cell cycle, Cell Tissue Kinet. 3 (1970) 321-334.

[10] C. Colijn and M. C. Mackey, Bifurcation and Bistability in a Model of Hematopoietic Regulation, SIAM J. Appl Dyn. Sys., Vol. 6, No. 2, (2007) 378-394.

[11] Diekmann O., Van Giles S., Verduyn Lunel S. and Walter H., Delay equations. Springer-Verlag, New-York (1995).

[12] T. Faria et M. L. Magalhaes, Normal Form for Retarded Functional Differential Equations with Parameters and Applications to Hopf Bifurcation, J. D. E. 122 (1995) 181-200.

[13] J. J. Ferrell, Tripping the switch fantastic : How protein kinase cascade convert graded into switch-like outputs, TIBS 21 (1996) 460-466.

[14] P. Fortin and M. C. Mackey, Periodic chronic myelogenous leukemia : spectral analysis of blood cell counts and etiological implications, Brit. J. Haematol. 104 (1999) 336-345.

[15] A. C. Fowler and M. C. Mackey, Relaxation oscillations in a class of delay differential equations, SIAM J. Appl. Math. Vol. 63 No. 1 (2003) 299-323.

[16] J.K.Hale and S. M. Verduyn Lunel, Introduction to functional Differential equations. Springer-Verlag, New-York (1993).

[17] B. D. Hassard, N. D. Kazarinoff et Y. H. Wan, Theory and Application of Hopf Bifurcation, Combridge University Press, London Mathematical Society, Lecture notes Series 41 (1981).

[18] C. Haurie, D. C. Dale and M. C. Mackey, Cyclical neutropenia and other periodic hematological disorders : A review of mechanisms and mathematical models, Blood 92 (1998) 2629-2640.

[19] C. Haurie, D. C. Dale, R. Rudnicki and M. C. Mackey, Mathematical modeling of complex neutrophil dynamics in the grey collie, J. Theor. Biol. 204 (2000) 505-519.

[20] C. Haurie, D. C. Dale and M. C. Mackey, Occurrence of periodic oscillations in the differential blood counts of congenital, idiopathic and cyclical neutropenic patients before and during treatment with G-CSF, Exper. Hematol. 27 (1999) 401-409.

[21] N. D. Hayes, Roots of the transcendental equation associated with a certain difference-differential equation. J. London Math. Soc. 25 (1950) 226-232.

[22] M. C. Mackey, Unified Hypothesis for the Origin of Aplastic Anemia and Periodic Hematopoiesis. Blood 51 (1978) 5.

[23] M. C. Mackey, Dynamic haematological disorders of stem cell origin, in Biophysical and Biochemical Information Transfer in Recognition, J. G. Vasseliva-Popova and E. V. Jensen, eds., Plenum publishing Corp., New York (1979) 373-409.

[24] M. C. Mackey, Mathematical Models of Haematopoietic Cell Replication and Control. In : The Art of Mathematical Modelling: Case Studies in Ecology, Physiology and Biofluids (Othmer, H. G., Adler, F. R., Lewis, M. A. and Dallon, J. C., eds), New York : Prentice-Hall (1997) 149-178.

[25] M. C. Mackey, Cell Kenitec Status of Haematopoietic Stem Cells. cell prolif. 34 (2001) 71-83.

[26] M. C. Mackey and P. Dormer, Continous maturation of proliferating erythroid precursors, Cell Tissue Kinet. 15 (1982) $381-392$.

[27] M. C. Mackey and R. Rudnicki, Global stability in a delayed partial differential equations describing cellular replication, J. Math. Biol. 33 (1994) 89-109.

[28] B. Macnamara, K. A. Palucka and A. Porwit-MacDonald, Balance between proliferation and apoptosis in leukemic cell lines resistant to cytostatics, Leuk. Lymphoma. 36 (1999) 179-189.

[29] J. M. Mahaffy, J. Bélair and M. C. Mackey, Hematopoietic model with moving boundary condition and state dependent delay, J. Theor. Biol. 190 (1998) 135-146.

[30] J. E. Marsden et M. McCracken, The Hopf Bifurcation and It's Application, Applied Math. Sciences, Springer -Verlag New York 19 (1976).

[31] H. Okita, A. Umezawa, M. Fukuma, T. Ando, F. Urano, M. Sano, Y. Nakata, T. Mori and J. Hata, Acute myeloid leukemia possessing jumping translocation is related to highly elevated levels of eat/mcl-1, a bcl-2 related gene with anti-apoptotic functions, Leuk. Res. 24 (2000) 73-77.

[32] Y. L. Ong M. F. McMullin, T. R. Lappin, F. G. Jones and A. E. Irvine, High bax expression is a good prognostic indicator in acute myeloid leukaemia, Br. J. Haematol. 111 (2000) 182-189.

[33] H. G. Othmer, F. R. Adler, M. A. Lewis and J. C. Dalton, eds., The art of mathematical modeling : Case studies in ecology, physiology and biofluids, Prentice Hall (1997).

[34] J. E. Parker, G. J. Mufti, F. Rasool, A. Mijovic, S. Devereux and A. Pagliuca, The role of apoptosis, proliferation, and the bcl-2-related proteins in the myelodysplastic syndromes and acute leukemia secondary to mds., Blood 96 (2000) 3932-3938.

[35] L. Pujo-Menjouet and M. C. Mackey, Contribution to the study of periodic chronic myelogenous leukemia, C. R. Biologies (2003). 
[36] L. Pujo-Menjouet, S. Bernard and M. C. Mackey, Long period oscillations in a $G_{0}$ model of hematopoietic stem cells, to appear.

[37] S. I. Rubinow and J. L. Lebowitz, A mathematical model of neutrophil production and control in normal leukemia, C. R. Biol. 1 (1975) 187-225.

[38] M. Santillan, J. M. Mahaffy, J. Bélair and M. C. Mackey, Regulation of platelet production : The normal response to perturbation and cyclical platelet disease, J. Theor. Biol. 206 (2000) 585-603.

[39] J. A. Smith and L. Martin, Do cells cycle?, Proc. Natl. Acad. Sci. USA 70 (1973) 1263-1267.

[40] J. Swinburune and M. C. Mackey, Cyclical thrombocytopenia : Characterization by spectral analysis and a review, J. Theor. Med. 2 (2000) 81-91.

[41] H. Talibi Alaoui and R. Yafia, Stability and Hopf bifurcation in Heamatopoietic Stem Cells Model, Electronic Journal of Differential Equations : Conference 11, pp. 167-173 (2004)

[42] H. Talibi Alaoui and R. Yafia, Stability and Hopf bifurcation in approchoable Heamatopoietic Stem Cells Model Mathematical Biosciences 206, pp 176-184 (2007).

[43] H. Talibi Alaoui, R. Yafia and M. A. Aziz Alaoui, Dynamics and Hopf Bifurcation Analysis In a Delayed Haematopoietic Stem Cells Model, Arab Journal of Mathematics and Mathematical Sciences. Vol. 1, No. 1, pp. 35-49 (2007).

[44] M. Wazewska-Czyzewska and A. Lasota, Mathematical problems of the dynamics of the red blood cell system, Mathematyka Stosowana 6, 23-40 (1976).

[45] G. Webb, Theory of nonlinear age-structured population dynamics, vol. 89, of monographs and textbooks in Pure and Appl. Math. Series, Dekker (1985). 